\title{
Deprescribing: What Is It and What Does the Evidence Tell Us?
}

Inappropriate prescribing and polypharmacy in older persons are associated with increased risks of falls, adverse drug events, hospital admissions, and death. ${ }^{1-3}$ Given these potential risks, it is imperative to find ways to manage the care of such

patients. One approach to handling unnecessary medication use and polypharmacy has been coined "deprescribing". Our recent presentation at the 2013 Professional Practice Conference of the Canadian Society of Hospital Pharmacists ${ }^{4}$ made it apparent that many pharmacists are unfamiliar with this concept. Given the consequences of inappropriate medication use in older patients, we believe it is an important topic to bring to light.

Deprescribing is the process of tapering, stopping, discontinuing, or withdrawing drugs, with the goal of managing polypharmacy and improving outcomes. Clinicians typically attempt to taper or stop agents on the basis of clinical experience and judgment, rather than using an approach guided by evidence. We recently set out to examine the evidence for deprescribing and to look for any published guidelines on the subject. We searched MEDLINE, Embase, OVID, International Pharmaceutical Abstracts, Google Scholar, and the bibliographies of identified articles.

The current body of evidence is well summarized by 2 reviews, ${ }^{5,6}$ although we found some additional articles not included in the reviews. Gnjidic and others ${ }^{5}$ summarized the results of randomized controlled trials involving deprescribing interventions. These authors focused on the interventions themselves (e.g., medication review by a pharmacist) rather than focusing on a specific drug or drug class. They found articles reporting the results of pharmacist- and physician-based interventions, as well as multidisciplinary approaches. The trials showed that deprescribing led to reductions in medication usage and cost; however, few of the studies investigated clinical outcomes. In studies that did examine outcomes, no differences were found in terms of patients' functional outcomes or in hospital admissions and mortality. Although these results may seem discouraging, we would point out that most of the trials likely had an insufficient duration of follow-up and insufficient power to detect clinically meaningful differences in outcomes. These problems underscore the need for well-designed, longterm studies that use clinical outcomes such as falls and deaths to assess the effects of deprescribing.

In a systematic review, Iyer and others ${ }^{6}$ identified about 30 randomized controlled trials and observational studies involving deprescribing. These researchers focused on specific drugs and drug classes and found mainly small, short-term studies that assessed the tolerability of deprescribing as opposed to clinical benefit. Overall, most of the trials showed that medications could be successfully withdrawn with little to no harm to the patient; some trials also demonstrated benefits such as reduced fall risk. ${ }^{7}$ Our literature search yielded some additional studies, but again, these had small samples with limited follow-up.

The results presented by Iyer and others ${ }^{6}$ are promising, but the difficulty lies in applying this evidence to patients in practice. The studies outlined in the reviews summarized above did not contain detailed methods indicating precisely how the medications were stopped. In fact, few studies described exactly how tapering of drugs was performed or provided detailed information about monitoring. As such, it would be difficult for practitioners to apply this evidence in practice. 
Our literature search revealed some guides and algorithms for deprescribing, but the approaches were described only in general terms and focused more on when or why a medication should be stopped rather than how. Garfinkel and others ${ }^{8}$ tested their deprescribing algorithm in a prospective cohort study and found that the rate of discontinuation failure was only $18 \%$ and mortality was significantly reduced at 1 year when the algorithm was applied. Again, however, little information was provided to describe exactly how medications were stopped.

We had difficulty finding a single search term that retrieved all relevant trials. Iyer and others ${ }^{6}$ also encountered this difficulty, noting that studies used a variety of terms, such as "cessation" and "discontinuation". The term "deprescribing", while not yet included as a MeSH (Medical Subject Heading) term, is useful because it is a unique and easily identifiable keyword that encompasses and unifies all of the various terms used in the literature (e.g., withdrawal, cessation). ${ }^{6}$

All available evidence considered, it is apparent that deprescribing in older persons likely results in reduced medication usage and cost and is unlikely to cause harm to patients. However, there is a lack of high-quality, long-term, prospective evidence to show that deprescribing results in clinically meaningful outcomes. Furthermore, the current body of evidence yields little guidance for practitioners on exactly how to deprescribe.

Given the risks of polypharmacy and inappropriate medication use in older persons, it is apparent that we need a larger, focused evidence base to guide deprescribing. Any studies intended to contribute to such an evidence base should include specific and systematic details of how medications were stopped to guide practitioners in the future.

\section{References}

1. Hajjar ER, Cafiero AC, Hanlon JT. Polypharmacy in elderly patients. Am J Geriatr Pharmacother. 2007;5(4):345-51.
2. Ziere G, Dieleman JP, Hofman A, Pols HAP, Van Der Cammen TJ, Stricker BHC. Polypharmacy and falls in the middle age and elderly population. $\mathrm{Br} \mathrm{J}$ Clin Pharmacol. 2006;61(2):218-23.

3. Jyrkkä J, Enlund H, Korhonen MJ, Sulkava R, Hartikainen S. Polypharmacy status as an indicator of mortality in an elderly population. Drugs Aging. 2009;26(12):1039-48.

4. Farrell B, Thompson W. The evidence for deprescribing: trials and tribulations [presentation]. Canadian Society of Hospital Pharmacists Professional Practice Conference; 2013 Feb 3-6; Toronto (ON).

5. Gnjidic D, Le Couteur DG, Kouladjian L, Hilmer SN. Deprescribing trials: methods to reduce polypharmacy and the impact on prescribing and clinical outcomes. Clin Geriatr Med. 2012;28(2):237-53.

6. Iyer S, Naganathan V, McLachlan AJ, Le Couteur DG. Medication withdrawal trials in people aged 65 years and older: a systematic review. Drugs Aging. 2008;25(12):1021-31.

7. Campbell AJ, Robertson MC, Gardner MM, Norton RN, Buchner DM. Psychotropic medication withdrawal and a home-based exercise program to prevent falls: a randomized, controlled trial. J Am Geriatr Soc. 1999;47(7):850-3.

8. Garfinkel D, Zur-Gil S, Ben-Israel J. The war against polypharmacy: a new costeffective geriatric-palliative approach for improving drug therapy in disabled elderly people. Isr Med Assoc J. 2007;9(6):430-4.

\author{
Wade Thompson, HBSC \\ Research Assistant \\ Barbara Farrell, BScPhm, PharmD, FCSHP \\ Scientist \\ Bruyère Research Institute \\ Ottawa, Ontario
}

Wade Thompson is also a student in the Bachelor of Science in Pharmacy program, School of Pharmacy, University of Waterloo, Waterloo, Ontario. Barbara Farrell is also Assistant Professor in the Department of Family Medicine, University of Ottawa, Ottawa, Ontario; Adjunct Assistant Professor in the School of Pharmacy, University of Waterloo, Waterloo, Ontario; and Clinical and Research Coordinator in the Pharmacy Department and a Pharmacist in the Geriatric Day Hospital of Bruyère Continuing Care, Ottawa, Ontario.

Competing interests: None declared.

\section{ON THE FRONT COVER}

\section{Balance Rock at Skidegate Haida Gwaii, British Columbia}

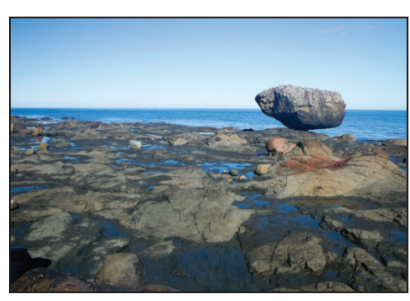

This photograph was taken near Skidegate on Haida Gwaii (formerly known as the Queen Charlotte Islands), British Columbia, on Aug 31, 2012. Haida Gwaii is a series of islands located at the most westerly point of northern British
Columbia. The image was captured by Monica Lee, a CSHP member, using a Canon 7D digital camera. It depicts Balance Rock, a 2-metre wide boulder deposited by glacial retreat. The rock has remained in place after recent earthquakes in the area.

The CJHP would be pleased to consider photographs featuring Canadian scenery taken by CSHP members for use on the front cover of the journal. If you would like to submit a photograph, please send an electronic copy (minimum resolution $300 \mathrm{dpi}$ ) to Colleen Drake at cdrake@cshp.ca. 